\title{
Carbon nanofibers prepared from electrospun polyimide, polysulfone and polyacrylonitrile nanofibers by ion-beam irradiation
}

\author{
Katsuya Sode ${ }^{1,2}$, Tomoki Sato ${ }^{1,2}$, Manabu Tanaka ${ }^{1,2}$, Yoshiaki Suzuki ${ }^{2}$ and Hiroyoshi Kawakami ${ }^{1,2}$ \\ Novel electrical conductive carbon nanofibers were prepared by a combination of electrospinning and ion-beam irradiation \\ techniques. Four types of polymer nanofibers, including polyimides (2,2'-bis(3,4-dicarboxyphenyl)hexafluoropropane \\ dianhydride (6FDA)-2,2'-bis(4-aminophenyl) hexafluoropropane (6FAP) and 6FDA-Bis(4-(4-aminophenoxy)phenyl) sulfone \\ (APPS)), polysulfone (PSF) and polyacrylonitrile, were prepared by the electrospinning method, which yielded fine nanofibers \\ with diameters of $\sim 150 \mathrm{~nm}$ (except PSF) under optimized conditions. The obtained polymer nanofibrous membranes \\ were irradiated with $\mathrm{Kr}^{+}$using an ion implanter at various ion fluences to give carbonized nanofibers. The electrical \\ conductivities of the carbon nanofibers increased with an increase in the ion fluence; the conductivity of the electrospun \\ 6FDA-6FAP nanofibrous membrane reached up to $c a .10^{-1} \mathrm{~S} \mathrm{~cm}^{-1}$ with $10^{16}$ ion cm $\mathrm{cm}^{-2}$ of $\mathrm{Kr}^{+}$. The influence of the starting \\ polymers on the electrical conductivity of the carbon nanofibers was studied by Raman and X-ray photoelectron spectroscopy analyses. \\ Polymer Journal (2013) 45, 1210-1215; doi:10.1038/pj.2013.56; published online 19 June 2013
}

Keywords: carbon nanofibers; conductivity; electrospinning; ion-beam irradiation

\section{INTRODUCTION}

Recently, much effort has been devoted to producing novel electrode materials for flexible and lightweight electronic devices. ${ }^{1,2}$ Carbon nanomaterials, such as carbon nanotubes, carbon nanofibers, graphene sheets and graphite powders, which possess excellent electrical conductivity and high mechanical strength, are possible candidates for electrode materials. ${ }^{3-5}$ However, the cost of these materials can be expensive, and their formability for use in electronic devices is often poor. Conventional carbon fibers, which are prepared by the carbonization of polymer precursors such as polyacrylonitrile (PAN) fibers at high temperature, can be obtained inexpensively; however, these carbon fibers typically have diameters ranging from 1 to $100 \mu \mathrm{m}$ and poor mechanical strength (rigid but brittle). ${ }^{6,7}$ As an alternative to microfibers, nanofibers prepared by applying an electrically charged jet to a polymer solution/melt (electrospinning) have recently received much attention. The rapidly developing technique of electrospinning provides a cost-effective approach to preparing fibers with nanometer-scale diameters for application in electronic components and devices. ${ }^{8-12}$ We have succeeded in preparing uniform non-beaded nanofibers with diameters of $\sim 30 \mathrm{~nm}$ by controlling the processing parameters, including applied voltage, feed rate, electrospinning humidity, and polymer solution viscosity and electrical conductivity. ${ }^{13,14}$ The productivity of nanofibers by the electrospinning method is sufficiently high to supply industrial applications by using non-nozzle type spinnerets and/or rotary drum collectors. The configuration of nanofiber assembly can also be controlled from nonwoven nanofibrous membranes to aligned nanofibers. ${ }^{8}$ We have reported novel uniaxially and biaxially aligned fluorinated polyimide nanofibers using specially designed collectors. ${ }^{15,16}$ Most recently, we first attempted the preparation of electrospun polyimide nanofibers for electrical conductive materials by a carbonization method using ion-beam irradiation. ${ }^{17}$

Ion-beam irradiation is a well-established technique for the postprocessing of materials and has been recognized as an effective method for the synthesis, modification and fabrication of materials, including polymers. ${ }^{18}$ The properties of polymers modified by ion irradiation or implantation strongly depend on the chemical structure and packing state of the polymer, as well as the type and energy of the incident ions. As irradiated ions penetrating into a polymer material lose energy by consecutive ion-polymer interactions, there is a gradual modification in the structure and morphology of the surface of the polymer material. For the past decade, we have reported that a series of asymmetric polyimide membranes, with ultrathin- and defect-free carbonized skin layers formed by the modification of membrane surfaces using ion-beam irradiation, show high gas permeability and selectivity. ${ }^{19-23}$ In our previous preliminary study, we demonstrated that ion-beam irradiation of electrospun polyimide (2,2'-bis(3,4-dicarboxyphenyl)hexafluoropropane dianhydride (6FDA)-2,2'-bis(4-aminophenyl) hexafluoropropane (6FAP)) nanofibers gave electrical conductive carbon nanofibers. ${ }^{17}$ Although 


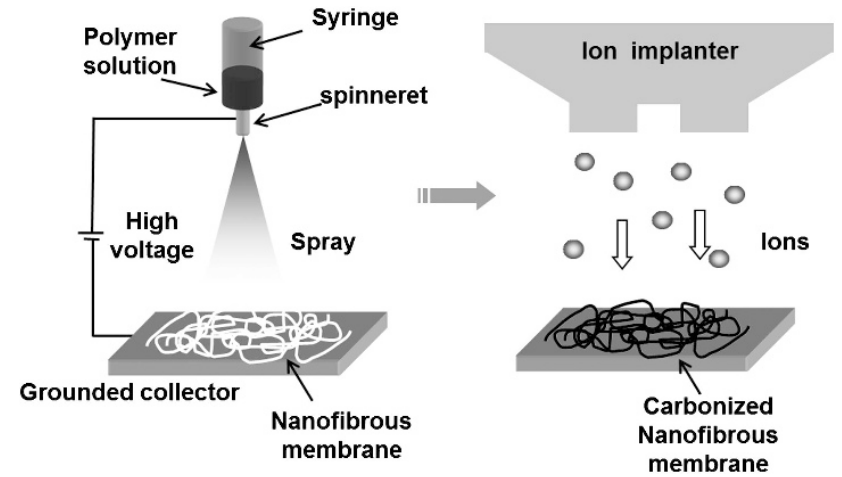

Figure 1 Schematic representation of electrospinning and ion-beam irradiation. A full color version of this figure is available at Polymer Journal online.

both electrospinning and ion-beam irradiation have been widely studied, our previous study was the first report on the combination of electrospinning and ion-beam irradiation (Figure 1). This new approach to the preparation of carbon nanofibers has several advantages over the conventional thermal carbonization method. ${ }^{24,25}$ This approach enabled accurate control over nanometer-scale fiber diameter by adjusting the electrospinning parameters and direct control over the carbon structure, either graphitic or amorphous, on the nanofiber surface by changing the ion-beam irradiation conditions. In addition, ion-beam-irradiated nanofibrous membranes had excellent formability and workability owing to the flexibility of the remaining flexible polymer nanofibrous layers under the carbonized surface. These flexible and cost-effective carbonized nanofibers are promising materials for future electronic devices.

To generalize this approach and to obtain knowledge for higher conductive carbon nanofibers, the influence of the starting polymers on the electrical conductivity of carbon nanofibers is studied in this paper by preparing four types of polymer nanofibers, including polyimides (6FDA-6FAP and 6FDA-Bis(4-(4-aminophenoxy)phenyl) sulfone (APPS)), polysulfone (PSF) and PAN. We chose polyimides and PSF for several reasons: they easily provide nanofibers by the electrospinning method ${ }^{13-16}$ and have distinctive thermal and mechanical properties that may help to maintain the nanofiber structures after ion-beam irradiation. ${ }^{17}$ PAN was chosen because there have been many reports on carbon fibers prepared from PAN fibers by thermal carbonization. ${ }^{6,7,24}$ Here, we discuss the morphology and conductivity differences among these electrospun polymer nanofibers formed via ion-beam irradiation at various ion fluences.

\section{EXPERIMENTAL PROCEDURE}

\section{Materials}

6FDA and 6FAP were purchased from Central Glass (Saitama, Japan). 6FDA was purified by sublimation before use. 6FAP was recrystallized twice from an ethanol solution before use. APPS was purchased from TCI (Tokyo, Japan) and purified by recrystallization from dichloromethane. PSF $\left(M_{\mathrm{w}}=6.4 \times 10^{4}\right)$ was obtained from BASF Japan (Tokyo, Japan) and used as received. PAN $\left(M_{\mathrm{w}}=1.5 \times 10^{5}\right)$ was purchased from Sigma-Aldrich (St Louis, MO, USA) and used without purification. Anhydrous $\mathrm{N}, \mathrm{N}$-dimethylformamide and anhydrous $N, N$-dimethylacetoamide were purchased from Kanto Chemical (Tokyo, Japan) and used as received.

Synthesis and characterization of 6FDA-6FAP and 6FDA-APPS

Polyimide 6FDA-6FAP was synthesized by chemical imidization of poly (amic acid) precursors, as reported in the literature. ${ }^{26}$ Another polyimide, 6FDAAPPS, was also synthesized using APPS as a monomer instead of 6FAP. The chemical structure of each polymer was characterized by proton nuclear magnetic resonance spectroscopy (B-500, Bruker, Germany). The molecular weights $\left(M_{\mathrm{w}}\right.$ and $\left.M_{\mathrm{n}}\right)$ of 6FDA-6FAP and 6FDA-APPS were determined by gel permeation chromatography (detector: Jasco 830-RI, JASCO, Tokyo, Japan), with tetrahydrofuran as the solvent at a flow rate of $1.0 \mathrm{ml} \mathrm{min}^{-1}$. The molecular weights were estimated by comparing the retention time on a column (KF-805L, Shodex, Tokyo, Japan) with those of standard polystyrene.

\section{Nanofiber preparation}

Polymers were separately dissolved in $N, N$-dimethylformamide ( $N, N$-dimethylacetoamide in the case of PSF) in an appropriate concentration. Each polymer solution was charged into a glass syringe equipped with a metal spinneret set for the electrospinning apparatus (ES-1000, Fuence, Saitama, Japan). Electrospinning conditions, such as the applied voltage, feeding rate of the polymer solution, distance between the spinneret and grounded collector plate and relative humidity in the apparatus, were carefully investigated to produce consistently uniform nanofibers. Finally, vacuum drying was carried out at $150^{\circ} \mathrm{C}$ for $10 \mathrm{~h}$ to remove the residual solvent from the fabricated nanofibers. Nanofiber morphology was evaluated using a scanning electron microscope (SEM; JXP-6100P, JEOL, Tokyo, Japan). To determine the nanofiber diameter, at least 5 photographs and 25 nanofibers were evaluated.

\section{Ion-beam irradiation}

Ion-beam irradiation was carried out on a $2 \times 2-\mathrm{cm}^{-2}$ surface area on the polymer nanofibrous membranes by a Riken ion implanter (Riken, Saitama, Japan). In this study, $\mathrm{Kr}^{+}$was used as the ion species, and ion irradiation was carried out at energy of $150 \mathrm{keV}$, ion-current density of $0.5 \mu \mathrm{A} \mathrm{cm}{ }^{-2}$ and a fluence ranging from $1 \times 10^{15}$ to $1 \times 10^{16}$ ions $\mathrm{cm}^{-2}$. The ion-beam currents were monitored by a Faraday cup, and the fluences were controlled by the beam current and irradiation time. Chemical structural changes after ion-beam irradiation were characterized by Raman spectroscopy (LabRam, Jobin Yvon, Tokyo, Japan) and X-ray photoelectron spectroscopy (XPS; ESCA3400, Shimadzu, Kyoto, Japan). Sixty-four scans of $2 \mathrm{~cm}^{-1}$ resolution were averaged to achieve a sufficient signal-to-noise ratio. The Raman spectra were observed using a He-Ne laser with a wavelength of $632.82 \mathrm{~nm}$. For the XPS measurement, after a wide scan from 0 to $1100 \mathrm{eV}$, narrow scans were carried out for 4 , 16,16 and 16 times on $\mathrm{C} 1 \mathrm{~s}, \mathrm{O} 1 \mathrm{~s}, \mathrm{~N}$ 1s and $\mathrm{F}$ 1s regions, respectively, with $\mathrm{X}$-ray conditions of $\mathrm{Mg}-\mathrm{K} \alpha, 10 \mathrm{~mA}$ and $10 \mathrm{kV}$.

\section{Measurement of electrical conductivity}

Electrical conductivity of electrospun nanofibrous membranes was evaluated by the four-probe method using a resistance meter (Loresta GP MCP-T610, Mitsubishi Chemical Analytech, Tokyo, Japan) at room temperature. The resistance values measured by the four-probe resistance meter were converted to electrical conductivity by considering the thickness of the practical conducting layer to be the depth of incoming irradiating $\mathrm{Kr}^{+}$into the polymers.

\section{TRIM simulation}

Transport of ions in matter (TRIM) 98 (IBM Research, New York, NY, USA) was used for the depth profile simulation of irradiated ions into polymers. ${ }^{27}$ The following simulation conditions were used: the ion species and energy were $\mathrm{Kr}^{+}$and $150 \mathrm{keV}$, respectively; the elemental composition and density of each polymer were input as target layer information; the depth of the target was set at $40000 \AA$; and the number of ions to calculate was 30000 . After the calculations, the nuclear and electronic energy losses were plotted as a function of depth.

\section{RESULTS AND DISCUSSION}

\section{Preparation of electrospun nanofibers}

Fluorinated polyimides, 6FDA-6FAP and 6FDA-APPS (Figure 2), were synthesized by chemical imidization consisting of treatment with a mixture of acetic anhydride and triethylamine. The degree of imidization of both polyimides was estimated to be almost $100 \%$ by proton nuclear magnetic resonance measurements. The synthesized 6FDA-6FAP and 6FDA-APPS were soluble in 
<smiles>CC(C)(C)Oc1ccc(C(C)(C)c2ccc(C(C)(C)c3ccc(N4C(=O)c5ccc(C(C)(C)C(F)(F)F)cc5C4=O)cc3)cc2)cc1</smiles><smiles>CN1C(=O)c2ccc(C(F)(F)C(F)(F)F)cc2C1=O</smiles>

6FDA-APPS<smiles>CC(C)(C)CC(C=N)C(C)(C)C</smiles>

PAN

Figure 2 Chemical structures of 2,2'-bis(3,4-dicarboxyphenyl)hexafluoropropane dianhydride (6FDA)-2,2'-bis(4-aminophenyl) hexafluoropropane (6FAP), 6 FDA-APPS, polysulfone (PSF) and polyacrylonitrile (PAN).

Table 1 Optimized electrospinning parameters and obtained nanofiber diameters

\begin{tabular}{lcccc}
\hline Polymer & Solvent & Concentration (wt\%) & Voltage (kV) & Fiber diameter (nm) \\
\hline 6FDA-6FAP & DMF & 12 & 20 & $159 \pm 19$ \\
6FDA-APPS & DMF & 13 & 20 & $214 \pm 34$ \\
PSF & DMAc & 29 & 24 & $359 \pm 128$ \\
PAN & DMF & 12 & 20 & $169 \pm 33$ \\
\hline
\end{tabular}

Abbreviations: DMAc, $N, N$-dimethylacetoamide; DMF, $N, N$-dimethylformamide; 6FAP, 2,2'bis(4-aminophenyl) hexafluoropropane; 6FDA, 2,2'-bis(3,4-dicarboxyphenyl)hexafluoropropane dianhydride; PAN, polyacrylonitrile; PSF, polysulfone

dianhydride; PAN, polyacrylonitrile; PSF, polysulfone. the collector, $10 \mathrm{~cm}$; relative humidity, $5 \% \mathrm{RH}$.

$N, N$-dimethylformamide, $N, N$-dimethylacetoamide , dimethylsulfoxide and $N$-methylpyrrolidone, and possessed high molecular weights with a narrow polydispersity index (6FDA-6FAP: $M_{\mathrm{w}}=3.8 \times 10^{5}$, $M_{\mathrm{w}} / M_{\mathrm{n}}=1.8 ; 6$ FDA-APPS: $\left.M_{\mathrm{w}}=2.3 \times 10^{5}, M_{\mathrm{w}} / M_{\mathrm{n}}=2.1\right)$.

The electrospinning process is a method of discharging a polymer solution in air from a spinneret under high voltage and producing nanofibers by exploiting the electrostatic repulsion of the polymer solution (Figure 1). It has been reported that the shape of the initiating droplet through the spinneret can be changed by the electrospinning conditions, including applied voltage, polymer solution viscosity and feeding rate, the distance between the spinneret and the grounded plate collector, and relative humidity in the apparatus. In particular, significant morphological changes were found when the concentration of the polymer solution was changed, indicating that the concentration or viscosity of the polymer solution is one of the most effective variables for controlling nanofiber morphology. We have reported that electrospun 6FDA-6FAP nanofibers obtained from 10 to $14 \mathrm{wt} \% \mathrm{~N}, \mathrm{~N}$-dimethylformamide or $\mathrm{N}, \mathrm{N}$-dimethylacetoamide solutions of 6FDA-6FAP were ultrafine, non-beaded and uniform, with diameters $<200 \mathrm{~nm}$ and narrow diameter distributions $(<20 \%)$. The other appropriate electrospinning conditions, including applied voltages, feeding rate, distance between the spinneret and the collector, and relative humidity, were $15-30 \mathrm{kV}, 0.12-1.2 \mathrm{ml} \mathrm{hr}^{-1}$, $10-20 \mathrm{~cm}$ and $5-10 \%$ relative humidity, respectively. ${ }^{13,14,16,17}$ It was also found that using a polymer concentration that was too low or too high gave mixtures of nanofibers and beads or thick fibers, respectively. On the basis of our knowledge, the optimal electrospinning conditions for 6FDA-APPS, PSF and PAN were

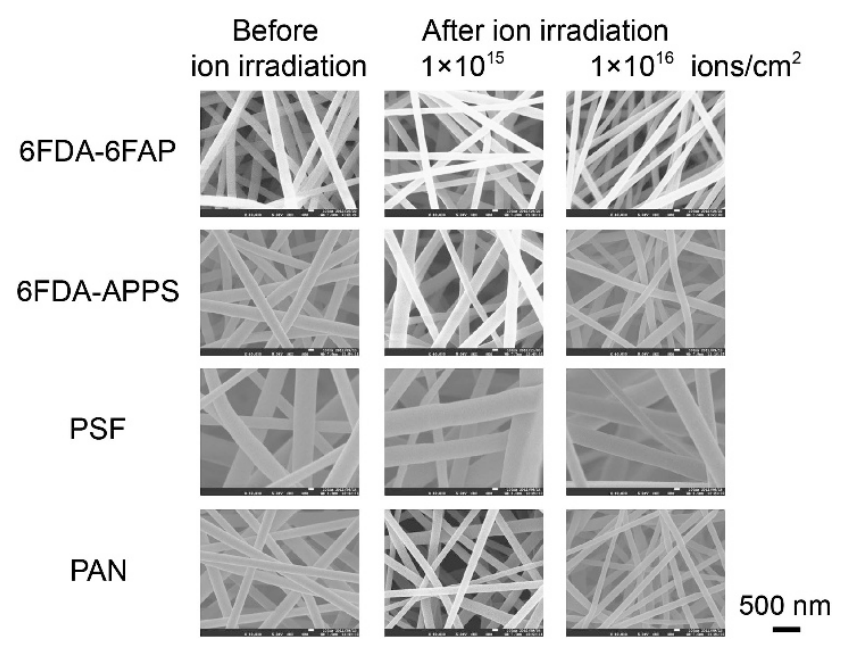

Figure 3 Scanning electron microscope (SEM) images of electrospun polymer nanofibers before and after $\mathrm{Kr}^{+}$irradiation.

carefully studied. Table 1 summarizes the optimized electrospinning conditions for each polymer. The SEM images of these polymer nanofibers are shown in the left column of Figure 3. All of the electrospun nanofibers were uniform, without any beads and the mean diameters of 6FDA-6FAP, 6FDA-APPS, PSF and PAN were $159 \pm 19,214 \pm 34,359 \pm 128$ and $169 \pm 33 \mathrm{~nm}(n=25)$, respectively, (Table 1). All the electrospun polymer nanofibers, except PSF, had fine diameters and narrow diameter distributions. The PSF nanofibers had relatively thick diameters; owing to its relatively low molecular weight, which was one order of magnitude less than the other polymers, a highly concentrated PSF solution was required to obtain sufficient viscosity for electrospinning. The diameter distribution of PSF nanofibers was wider than that of other polymer nanofibers.

This result is also explained by the lower molecular weight of PSF, which results in less polymer chain entanglement and a lack of stability during the electrospinning process.

\section{Ion-beam irradiation}

Ion-beam irradiation is a physicochemical surface modification process resulting from the impingement of a high-energy ion beam. The ion depth through the polymers was regulated by changing the ion species and its energy. In this study, ion irradiation was performed 

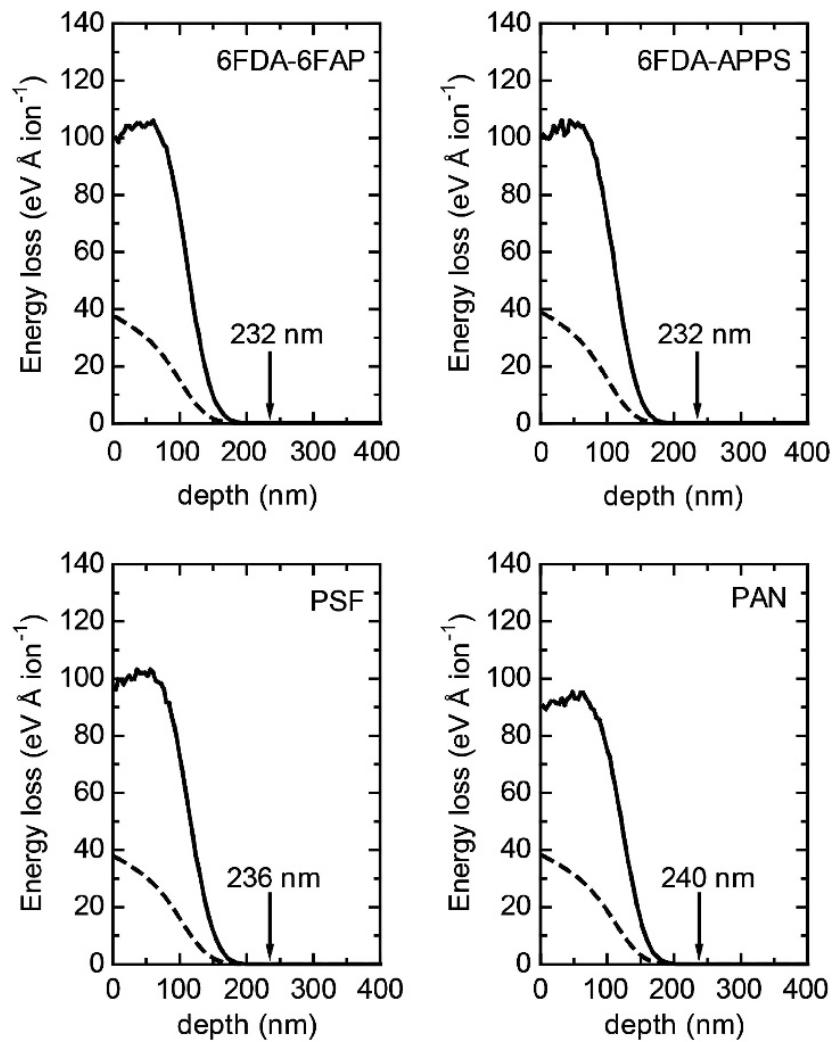

Figure 4 Relationship between ion energy loss and ion depth in $2,2^{\prime}$ bis(3,4-dicarboxyphenyl)hexafluoropropane dianhydride (6FDA)-2,2' bis(4-aminophenyl) hexafluoropropane (6FAP), 6FDA-APPS, polysulfone (PSF) and polyacrylonitrile (PAN), as calculated from the transport of ions in matter (TRIM) simulation. Solid line, nuclear energy loss; dashed line, electronic energy loss.

on the electrospun polymer nanofibrous membranes, and $\mathrm{Kr}^{+}$was chosen as the ion species based on our previous study, where the electrical conductivity of nanofibers increased with the ion-beam irradiation in the order of $\mathrm{He}^{+}<\mathrm{Ne}^{+}<\mathrm{Ar}^{+} .{ }^{17}$ Although heavier ion spices were more effective in increasing the electrical conductivities of the nanofibers, the ion depth through the polymer decreased with increasing atomic number. In this study, therefore, $\mathrm{Kr}^{+}$that is heavier than previously used $\mathrm{Ar}^{+}$was chosen, with consideration of its ion depth and the nanofiber diameters, as described later. The electrospun polymer nanofibrous membranes were put in the ion implanter and were irradiated by $150 \mathrm{keV} \mathrm{Kr}^{+}$at an ion-current density of $0.5 \mu \mathrm{Acm}^{-2}$ and ion fluences of $1 \times 10^{15}, 5 \times 10^{15}$ or $1 \times 10^{16}$ ions $\mathrm{cm}^{-2}$. Irradiating ions penetrating into the electrospun polymer nanofibers lose energy through several ion-polymer interactions. One such energy loss is electronic, involving the processes of electronic excitation and ionization of the atoms in the polymers, which arise from exchange of electrons between the incident ions and the atoms in the polymer. Another energy loss is nuclear, involving atomic collisions between the incident ions and the atoms in the polymer. The depth profile of the energy loss for $\mathrm{Kr}^{+}$irradiation at $150 \mathrm{keV}$ in each polymer was estimated using the well-established Monte Carlo simulation method (TRIM code). From the TRIM simulation, $150 \mathrm{keV} \mathrm{Kr}^{+}$-irradiating ions in 6FDA-6FAP, 6FDA-APPS, PSF and PAN completely lose their electronic and nuclear energies at depths of 232, 232, 236 and $240 \mathrm{~nm}$, respectively, (Figure 4).
The morphological change of the electrospun nanofibers after ion irradiation was characterized by SEM. Figure 3 depicts SEM images of the electrospun polymer nanofibers before and after ion-beam irradiation at various ion fluences. The nanofibers after ion-beam irradiation also have a flat and smooth external appearance similar to that of the nonirradiated pristine nanofibers. As ion-beam irradiation is a surface modification method unlike thermal treatments at high temperatures, damage on the nanofibers is limited. Although the surfaces of the fiber mats turned black, the nanofibrous membranes still maintained their flexibility after the ion-beam irradiation. The SEM images of the nanofibers after $150 \mathrm{keV} \mathrm{Kr}^{+}$irradiation at $1 \times 10^{16}$ ions $\mathrm{cm}^{-2}$ revealed that the nanofiber diameters were slightly reduced to $131 \pm 19$, $158 \pm 25,341 \pm 192$ and $141 \pm 29 \mathrm{~nm}$ for 6FDA-6FAP, 6FDA-APPS, PSF and PAN nanofibers, respectively. The decreasing rates of diameters after ion-beam irradiation were $\sim 18 \%, 26 \%, 5 \%$ and $17 \%$ for 6 FDA6FAP, 6FDA-APPS, PSF and PAN nanofibers, respectively. Carbonization likely occurs in these polymer nanofibers, and non-carbon elements, such as fluorine and nitrogen, were desorbed from the nanofibers by ion irradiation to reduce their diameters.

\section{Electrical conductivity}

The electrical conductivities of the electrospun nanofibrous membranes were evaluated by the four-probe method using a resistance meter. The thicknesses of obtained electrospun nanofibrous membranes were several micrometers; however, ion irradiation was carried out only on the nanofibers located near the surface of the nanofibrous membrane. As the bottom layers consist of nonirradiated pristine polymer nanofibers that have low electrical conducting properties, the practical conducting layer consisted of the ion-irradiated layers. The surface resistivities ( $\Omega$ per square) measured by the four-probe resistance meter were converted to apparent electrical conductivities $\left(\mathrm{S} \mathrm{cm}^{-1}\right)$ by using the thickness of the practical conducting layer, that is, the depth of incoming irradiated $\mathrm{Kr}^{+}$into the polymers (for example, $232 \mathrm{~nm}$ for 6FDA-6FAP, from the TRIM simulation described previously). The actual electrical conductivities of the nanofibrous membranes must be higher than the apparent values because of vacancies among the nanofibers. Although actual electrical conductivity can be calculated by considering porosity, connectivity and actual conductive path length, it is difficult to estimate the valid electrical conductivities. Therefore, in this paper apparent values are calculated from the surface resistivity and the thickness of the practical conducting layer, and are used as the reported electrical conductivities. The electrical conductivities of the electrospun polymer nanofibrous membranes after ion irradiation at various ion fluences are plotted in Figure 5. All of the electrospun polymer nanofibrous membranes showed no electrical conductivity $\left(\ll 10^{-6} \mathrm{~S} \mathrm{~cm}^{-1}\right)$ before ion irradiation. Once the 6FDA-6FAP nanofibrous membrane was irradiated by $\mathrm{Kr}^{+}$at $1 \times 10^{15}$ ion $\mathrm{cm}^{-2}$, conductivity $>10^{-3} \mathrm{~S} \mathrm{~cm}^{-1}$ was observed. The conductivity of the 6FDA-6FAP nanofibrous membrane increased with ion fluence and reached $\mathrm{ca} .10^{-1} \mathrm{~S} \mathrm{~cm}^{-1}$ with $1 \times 10^{16}$ ion $\mathrm{cm}^{-2}$. The 6FDA-APPS and PAN nanofibrous membranes showed lower conductivities than 6FDA-6FAP at all ranges of ion fluences. The conductivity of the 6FDA-APPS nanofibrous membrane $\left(c a .10^{-3} \mathrm{~S} \mathrm{~cm}^{-1}\right)$ was found to be reasonable when compared with reported conductivities of ionbeam-irradiated polyimide membranes with similar polymer structures $\left(10^{-2}-10^{-4} \mathrm{~S} \mathrm{~cm}^{-1}\right)^{28}$ by considering the differences in the ion irradiation conditions. PSF showed the lowest conductivity, that is, the conductivity of the PSF nanofibrous membrane was still $<10^{-3} \mathrm{Scm}^{-1}$, even after $\mathrm{Kr}^{+}$irradiation at an ion fluence of 


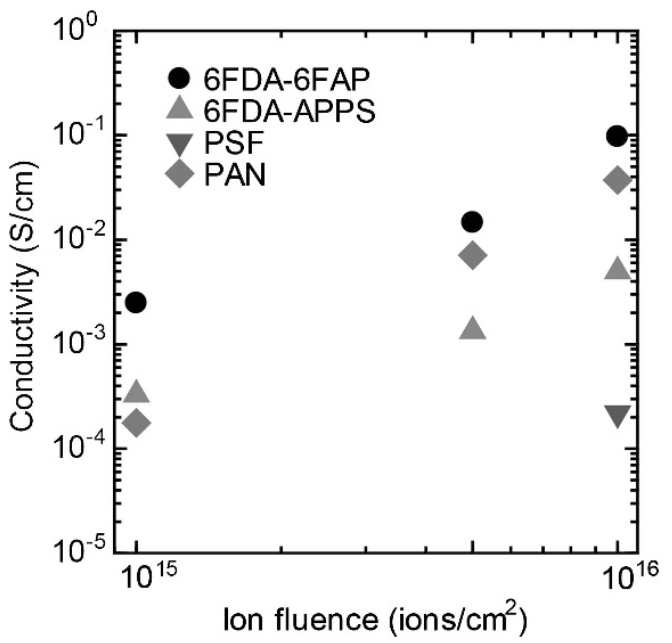

Figure 5 Electrical conductivities of ion-irradiated 2,2'-bis(3,4-dicarboxyphenyl)hexafluoropropane dianhydride (6FDA)-2,2'-bis(4-aminophenyl) hexafluoropropane (6FAP), 6FDA-APPS, polysulfone (PSF) and polyacryIonitrile (PAN) nanofibrous membranes as a function of ion fluence. A full color version of this figure is available at Polymer Journal online.

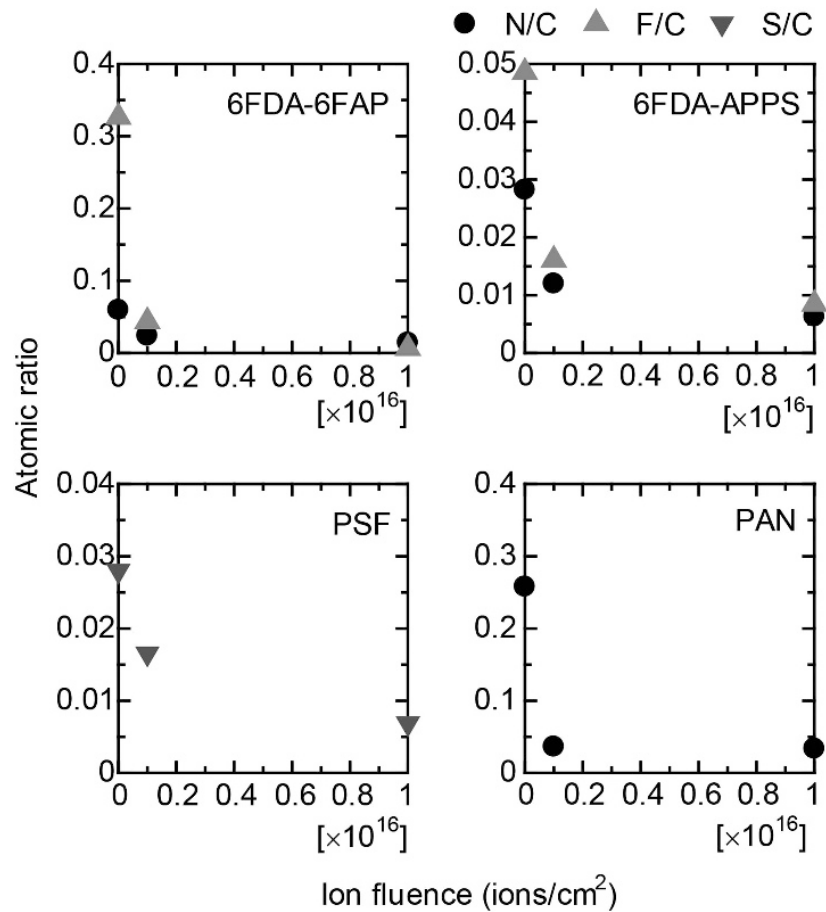

Figure $6 \mathrm{~N} / \mathrm{C}, \mathrm{F} / \mathrm{C}$ and S/C atomic ratios of ion-irradiated 2,2'-bis(3,4dicarboxyphenyl)hexafluoropropane dianhydride (6FDA)-2,2'-bis(4-aminophenyl) hexafluoropropane (6FAP), 6FDA-APPS, polysulfone (PSF) and polyacrylonitrile (PAN) nanofibrous membranes, as measured by X-ray photoelectron spectroscopy (XPS) spectroscopy. A full color version of this figure is available at Polymer Journal online.

$10^{16}$ ion $\mathrm{cm}^{-2}$, and was not detectable at ion fluences of $1 \times 10^{15}$ and

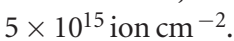

\section{XPS and Raman spectroscopy}

To understand their chemical structural changes, the elemental compositions of the polymer nanofibers were characterized by XPS. Figure 6 shows the atomic ratios (N/C, F/C and S/C) of the 6FDA-6FAP, 6FDA-
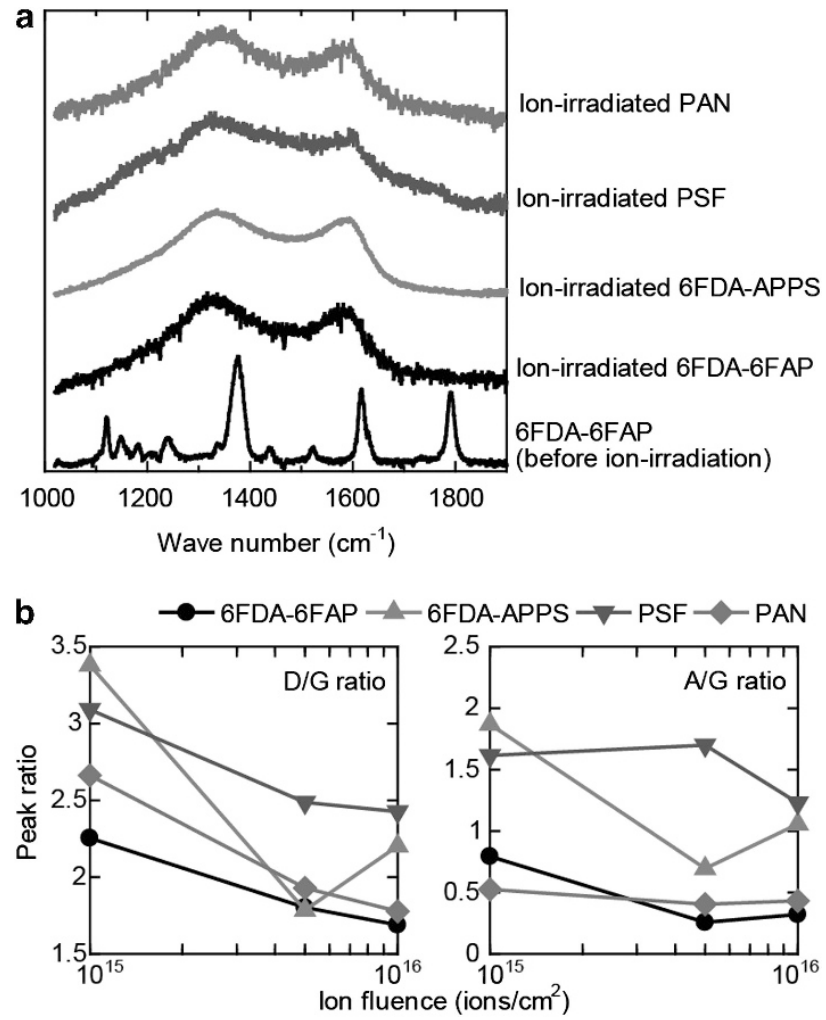

Figure 7 (a) Raman spectra of 2,2'-bis(3,4-dicarboxyphenyl)hexafluoropropane dianhydride (6FDA)-2,2'-bis(4-aminophenyl) hexafluoropropane (6FAP) and ion-irradiated 6FDA-6FAP, 6FDA-APPS, polysulfone (PSF) and polyacrylonitrile (PAN) nanofibrous membranes. (b) Disorder carbon (D)/graphitized carbon (G) and amorphous carbon (A)/G peak ratios of ionirradiated 6FDA-6FAP, 6FDA-APPS, PSF and PAN nanofibrous membranes, as measured by Raman spectroscopy. A full color version of this figure is available at Polymer Journal online.

APPS, PSF and PAN nanofibers estimated from the XPS spectra. All of the $\mathrm{N} / \mathrm{C}, \mathrm{F} / \mathrm{C}$ and $\mathrm{S} / \mathrm{C}$ values decreased with increasing ion fluences, indicating that carbonization via desorption of the non-carbon elements from the nanofibers occurred as a result of the ion irradiation. Carbonization was also confirmed by the Raman spectra of the polymer nanofibers before and after ion irradiation (fluence at $1 \times$ $10^{16}$ ions $\mathrm{cm}^{-2}$ ), as shown in Figure $7 \mathrm{a}$. The peaks at 1240, 1380, 1620 and $1780 \mathrm{~cm}^{-1}$ in the bottom spectrum of pristine 6FDA-6FAP nanofibers are assigned to $\mathrm{C}-\mathrm{F}, \mathrm{C}-\mathrm{N}$, and $\mathrm{C}=\mathrm{C}$ in the aromatic ring and $\mathrm{C}=\mathrm{O}$, respectively. After irradiation by $150 \mathrm{keV} \mathrm{Kr}^{+}$at a fluence of $1 \times 10^{16}$ ions $\mathrm{cm}^{-2}$, these characteristic peaks disappeared, and two broad peaks appeared at $\sim 1360$ and $1590 \mathrm{~cm}^{-1}$ on the irradiated nanofibers. These two broad peaks are known to correspond to the disorder carbon (D) and graphitized carbon (G) broad bands, respectively, indicating that the surface of the electrospun nanofiber changed to carbon-enriched disordered graphitic material. ${ }^{3}$ Similar phenomena were observed with the 6FDA-APPS, PSF and PAN nanofibrous membranes after ion-beam irradiation. The Raman spectral area ratio (peak ratio) between the $D$ and $G$ bands (D/G ratio), and between the amorphous carbon (A) band at $\sim 1500 \mathrm{~cm}^{-1}$ ) and $\mathrm{G}$ bands (A/G ratio) of these polymer nanofibrous membranes were plotted in Figure $7 \mathrm{~b}$. As decreasing $\mathrm{D} / \mathrm{G}$ and $\mathrm{A} / \mathrm{G}$ values imply graphitization, Figures 6 and 7 indicate that 6FDA-6FAP is more facile toward carbonization and graphitization than are other polymers. These XPS and Raman spectroscopy results are in agreement with the electrical conductivity trends of the electrospun nanofibrous membranes. 
Relationship between electrical conductivity and polymer structure Here, we organize the relationship between electrical conductivity and polymer structure. Among the four polymers, the PAN nanofibrous membranes showed a characteristic behavior in which conductivity was drastically increased with increasing ion fluence. This behavior is expected to be derived from the well-known formation of sixmembered triazine derivatives by the cyclization of nitrile groups. ${ }^{29}$ However, the electrical conductivities of the ion-irradiated polymer nanofibrous membranes were much lower than those of the reported carbon fibers prepared by thermal carbonization ${ }^{24}$ because of the difference in fiber diameters and the porosities of the nanofibrous membranes. The narrower fibers and higher porosity of the nanofibrous membrane must result in lower electrical conductivity because of the increased contact resistance between the nanofibers and the less conductive pathway from the presence of air among the nanofibers. On the other hand, fluorinated polyimides were favorable for carbonization and graphitization by ion-beam irradiation owing to their aromatic structures and the existence of labile leaving groups, such as trifluoromethyl groups. Compared with PSF, polyimide, such as 6FDA-6FAP, has flatter moieties composed of five- and sixmembered benzimide groups in the polymer backbones that facilitate the formation of carbon clusters upon ion irradiation. Meanwhile, ether and sulfone moieties in the PSF backbone may impede the continuity of the carbon clusters and subsequent restructuring after ion irradiation. 6FDA-APPS has both typical structures categorized for polyimide and PSF in its polymer backbone; therefore, it showed intermediate conductivity that was almost equivalent to the values reported for similar ion-beamirradiated membranes ${ }^{28}$ and intermediate degrees of carbonization and graphitization, as shown by Raman spectroscopy and XPS results. Consequently, we conclude that the electrical conductivities of ionirradiated polymer nanofibers, except those of the atypical PAN nanofibers, are related to the polymer structure, and polymers bearing labile leaving groups and a flatter backbone without several functional groups that impede the continuity of carbon clusters are favorable for carbon nanofibers with higher electrical conductivity.

\section{CONCLUSION}

In this study, we investigated the relationship between polymer structure and conductivity of electrospun polymer nanofibrous membranes after ion irradiation. Four polymer nanofibers, 6FDA-6FAP, 6FDA-APPS, PSF and PAN, were prepared by the electrospinning method and showed narrow diameters under the optimized conditions. Irradiation by $\mathrm{Kr}^{+}$at various fluences gave conductivities on the insulating electrospun polymer nanofibrous membranes reaching $10^{-4}-10^{-1} \mathrm{~S} \mathrm{~cm}^{-1}$. Raman spectroscopy and XPS studies supported the presence of carbonization and graphitization in these polymer nanofibers after ion irradiation. The conductivity enhancement by ion irradiation was varied for different polymer structures, which is explained by the existence of labile leaving groups, the planarity of polymer backbone and the continuity of carbon clusters. Although the fluorinated polyimide, 6FDA-6FAP, nanofibrous membranes showed higher electrical conductivities ( $c$ a. $10^{-1} \mathrm{~S} \mathrm{~cm}^{-1}$ ) than the other materials, the conductivity was not sufficient for practical applications. Ongoing work in our group includes studying the catalytic effect of additives on the electrical conductivities of electrospun nanofibrous membranes to obtain higher conductivities, and these optimized membranes can be utilized for flexible electronic applications.

\section{ACKNOWLEDGEMENTS}

This work was partially supported by a grant from the ALCA program of the Japan Science and Technology Agency.

1 Wong, W. S. \& Salleo, A. Flexible Electronics: Materials and Applications (Springer, New York, 2009)

2 Helander, M. G. Solution-processible electrodes. Science 336, 302-303 (2012).

3 McCreery, R. L. Advanced carbon electrode materials for molecular electrochemistry. Chem. Rev. 108, 2646-2687 (2008).

4 Liu, R., Duaya, J. \& Lee, S. B. Heterogeneous nanostructured electrode materials for electrochemical energy storage. Chem. Commun. 47, 1384-1404 (2011).

5 Hecht, D. S., Hu, L. \& Irvin, G. Emerging transparent electrodes based on thin films of carbon nanotubes, graphene, and metallic nanostructures. Adv. Mater. 23, 1482-1513 (2011).

6 Chen, J. C. \& Harrison, I. R. Modification of polyacrylonitrile (PAN) carbon fiber precursor via post-spinning plasticization and stretching in dimethyl formamide (DMF). Carbon. N. Y. 40, 25-45 (2002)

7 Ozkan, T., Naraghi, M. \& Chasiotis, I. Mechanical Strength of Vapor Grown Carbon Nanofibers. Carbon. N. Y. 48, 239-244 (2010)

8 Matsumoto, H. \& Tanioka, A. in Electrical Phenomena at Interfaces and Biointerfaces: Fundamentals and Applications in Nano-, Bio-, and Environmental Sciences: (ed. Ohshima, H.) Ch. 27, 649-680 (John Wiley \& Sons, New Jersey, 2012).

9 Dzenis, Y. Spinning continuous fibers for nanotechnology. Science 304, 1917-1919 (2004).

10 Thavasi, V., Singh, G. \& Ramakrishna, S. Electrospun nanofibers in energy and environmental applications. Energy Environ. Sci. 1, 205-221 (2008).

11 Long, Y. Z., Li, M. M., Gu, C., Wan, M., Duvail, J. L., Liu, Z. \& Fan, Z. Recent advances in synthesis, physical properties and applications of conducting polymer nanotubes and nanofibers. Prog. Polym. Sci. 36, 1415-1442 (2011).

12 Miao, J., Miyauchi, M., Simmons, T. J., Dordick, J. S. \& Linhardt, R. J. Electrospinning of nanomaterials and applications in electronic components and devices. J. Nanosci. Nanotech. 10, 5507-5519 (2010).

13 Fukushima, S., Karube, Y. \& Kawakami, H. Preparation of ultrafine uniform electrospun polyimide nanofiber. Polym. J. 42, 514-518 (2010).

14 Arai, T. \& Kawakami, H. Ultrafine electrospun nanofiber created from cross-linked polyimide solution. Polymer 53, 2217-2222 (2012).

15 Tamura, T. \& Kawakami, H. Aligned electrospun nanofiber composite membranes for fuel cell electrolytes. Nano. Lett. 10, 1324-1328 (2010).

16 Karube, Y. \& Kawakami, H. Fabrication of well-aligned electrospun nanofibrous membrane based on fluorinated polyimide. Polym. Adv. Tech. 21, 861-866 (2010).

17 Seki, N., Arai, T., Suzuki, Y. \& Kawakami, H. Novel polyimide-based electrospun carbon nanofibers prepared using ion-beam irradiation. Polymer 53, 2062-2067 (2012).

18 Kondyurin, A. \& Bilek, M. Ion Beam Treatment of Polymers: Application Aspects from Medicine to Space (Elsevier, Oxford, 2008).

19 Iwase, M., Sannomiya, A., Nagaoka, S., Suzuki, Y., Iwaki, M. \& Kawakami, H. Gas permeation properties of asymmetric polyimide membranes with partially carbonized skin layer. Macromolecules 37, 6892-6897 (2004).

20 Sannomiya, A., Nagaoka, S., Suzuki, Y., Iwaki, M. \& Kawakami, H. Gas diffusion and solubility in he+-irradiated asymmetric polyimide membranes. Polymer. 47, 6585-6591 (2006).

21 Sannomiya, A., Fukui, K., Nagaoka, S., Suzuki, Y., Iwaki, M. \& Kawakami, H. Gas transport properties of asymmetric polyimide membranes prepared by ion-beam irradiation. J. Polym. Sci. B. 45, 262-269 (2007).

22 Tezuka, T., Kobayashi, T., Muraoka, D., Nagaoka, S., Suzuki, Y. \& Kawakami, H. Gas transport properties of asymmetric polyimide membranes prepared by plasma-based ion implantation. Polym. Adv. Tech 20, 987-992 (2009).

23 Muraoka, D., Zhang, L., Suzuki, Y. \& Kawakami, H. Effects of iron catalyst on gas transport properties of ion-irradiated asymmetric polyimide membranes. J. Memb. Sci. 375, 75-80 (2011).

24 Che, A. F., Germain, V., Cretin, M., Cornu, D., Innocent, C. \& Tingry, S. Fabrication of free-standing electrospun carbon nanofibers as efficient electrode materials for bioelectrocatalysis. N. J. Chem. 35, 2848-2853 (2011).

25 Imaizumi, S., Matsumoto, H., Suzuki, K., Minagawa, M., Kimura, M. \& Tanioka, M. Phenolic resin-based carbon thin fibers prepared by electrospinning: additive effects of poly(vinyl butyral) and electrolytes. Polym. J. 41, 1124-1128 (2009)

26 Kawakami, H. Anzai, J. \& Nagaoka, S. Gas transport properties of soluble aromatic polyimides with sulfone diamine moieties. J. Appl. Polym. Sci. 57, 789-795 (1995).

27 Ziegler, J. F., Biersack, J. P. \& Littmark, W. The Stopping and Range of lons in Solids (Pergamon, New York, 1985).

28 Popok, V. N., Azarko, I. I., Khaibullin, R. I., Stepanov, A. L., Hnatowicz, V., Mackova, A. \& Prasalovich, S. V. Radiation-induced change of polyimide properties under high-fluence and high ion current density implantation. Appl. Phys. A. 78, 1067-1072 (2004).

29 Nabais, J. M. V., Carrott, P. J. M. \& Carrott, M. M. L. R. From commercial textile fibres to activated carbon fibres: chemical transformations. Mater. Chem. Phys. 93, 100-108 (2005). 